\section{Concepts in Pharmacogenomics}

Zdanowicz M M. American Society of Health-System Pharmacists, Bethesda, Maryland, 2010. Softcover, 414 pages. ISBN 978-158528-234-0. US\$69 (US\$62 for ASHP members).

Concepts in Pharmacogenomics is a combined textbook and practical guide intended to provide practising pharmacists and pharmacy trainees (undergraduate and graduate students, residents, fellows, etc.) with an overview of the basic science underlying pharmacogenomics, as well as details of relevant clinical applications and related issues.

The book is unique because it specifically targets pharmacists and pharmacy trainees. Although the book can be used as a primary textbook for either mandatory or elective courses in pharmacogenomics, practising pharmacists can use it in a self-study fashion.

Lead author Martin Zdanowicz has an MA in biology, a $\mathrm{PhD}$ in pharmacology, and extensive experience as a research scientist and pharmacy academician with research interests in pharmacogenomics, development of the pharmacy curriculum, and pharmacy education. He has garnered the assistance of 21 other contributing authors in writing the specific chapters. One contributor is a physician, and the others have a PharmD or $\mathrm{PhD}$, most of these being affiliated with a school of pharmacy. There were also 4 reviewers ( 2 with a PharmD and 2 with a $\mathrm{PhD})$. Although many of the authors are not "big names" in the field of pharmacogenomics, they are all (according to the book's preface) "individuals who used, taught, or had a thorough understanding of pharmacogenomics and the impact it could have in their particular area of expertise."

The book is divided into 3 parts:

- Part I, entitled "Fundamentals of Pharmacogenomics", encompasses 6 chapters: "Pharmacogenomics: Past, Present, and Future", "The Genetic Basis of Pharmacogenomics", "Methodologies in Pharmacogenomics", "The Pharmacogenetics of Drug Metabolism", "Pharmacogenomics and Drug Transport/Efflux", and "Pharmacodynamics and Pharmacogenomics". These chapters focus on the basic science aspects of pharmacogenomics, with an emphasis on methodology, pharmacokinetics, and pharmacodynamics.

- Part II, entitled "Applications of Pharmacogenomics in Therapeutics", also consists of 6 chapters: "Cardiovascular Disease", "Hematology/Oncology Pharmacogenomics", "Central Nervous System", "Infectious Diseases", "Respiratory Diseases", and "Toxigenomics". These chapters present a systems-based approach to the therapeutic applications of pharmacogenomics.

- Part III, "Important Issues in Pharmacogenomics", contains 5 chapters: "The Role of Pharmacists in Pharmacoge- nomics", "Pharmacogenomics and Clinical Testing", "Pharmacogenomics in Pharmacy Education", "Ethical Considerations in Pharmacogenomics", and "Future Promise of Pharmacogenomics in Clinical Practice".

Each chapter begins with specific learning objectives and a list of key definitions. The body of each chapter features case studies, clinical pearls, and summary tables and figures. All of this material is presented in a clear, organized, and user-friendly format and can be easily distinguished from the main text. The entire book is written clearly in language that will be understandable to practitioners and students alike.

In my opinion, this book has only 2 minor limitations. First, there is some inconsistency among chapters in the presentation of the clinical pearls. Some are very general, others very specific, and some are not what one would typically consider a "clinical pearl" (e.g., "Linkage disequilibrium is a basis for genetic association studies including genome-wide association studies"). These variations make it apparent to the reader that the chapters are written by different authors. The second limitation, as acknowledged in the book's preface, is that the field of pharmacogenomics is constantly evolving and rapidly advancing and the information contained in a volume like this one may become outdated shortly after (or even before) publication. Although the lead author envisions a companion website where the authors could post regular updates and other supplemental materials for their respective chapters, such a resource does not appear to be currently available. The last chapter, however, does contain a list of resources (i.e., journals and websites) which readers can use to keep up to date.

Overall, Zdanowicz and the contributing authors have filled a niche with this useful, easy-to-follow textbook and practical guide through which pharmacists and trainees alike can enhance their knowledge of pharmacogenomics. In addition, this book will help pharmacists to appreciate how their unique qualifications as well-recognized medication experts can help them to take a proactive and leading role in moving pharmacogenomics from a research tool to a clinical discipline.

Mary H H Ensom, PharmD, FASHP, FCCP, FCSHP, FCAHS

Professor and Director

Doctor of Pharmacy Program

Faculty of Pharmaceutical Sciences

Distinguished University Scholar

The University of British Columbia

Clinical Pharmacy Specialist

Children's and Women's Health Centre of British Columbia

Vancouver, British Columbia

Dr Ensom is also the Editor of the CJHP. 